\title{
Editorial
}

\section{New Developments on Robust Nonlinear Control and Its Applications}

\author{
Ming-Cong Deng, ${ }^{1}$ Hong-Nian Yu, ${ }^{2}$ Shu-Hui Bi, ${ }^{3}$ and Sheng-Jun Wen ${ }^{4}$ \\ ${ }^{1}$ Department of Electrical and Electronic Engineering, Tokyo University of Agriculture and Technology, 2-24-16 Nakacho, \\ Koganei, Tokyo 184-8588, Japan \\ ${ }^{2}$ School of Design, Engineering and Computing, Bournemouth University, Poole House P316, Talbot Campus, Poole BH12 5BB, UK \\ ${ }^{3}$ Shandong Provincial Key Laboratory of Robot and Manufacturing Automation Technology, Institute of Automation, \\ Shandong Academy of Sciences, 19 Keyuan Road, Jinan, Shandong 250064, China \\ ${ }^{4}$ Department of Electronic and Information Engineering, Zhongyuan University of Technology, Zhongyuan Road No. 41, \\ Zhengzhou 450007, China
}

Correspondence should be addressed to Ming-Cong Deng; deng@cc.tuat.ac.jp

Received 25 June 2014; Accepted 25 June 2014; Published 6 July 2014

Copyright (C) 2014 Ming-Cong Deng et al. This is an open access article distributed under the Creative Commons Attribution License, which permits unrestricted use, distribution, and reproduction in any medium, provided the original work is properly cited.

In the real industrial control systems, most of them are nonlinear systems. Also, it is difficult to obtain the exact model of the real controlled plant because the modeling is affected by many uncertain factors, such as change of system operation conditions, parameter uncertainties, faults, and other external disturbances. Therefore, the robust nonlinear control and design for the nonlinear systems received considerable attention and have been studied by many researchers in different fields.

In the last three decades, a variety of robust nonlinear control and design techniques have been proposed, such as PID control method [1], feedback linearization control method [2], sliding mode control method [3], adaptive control approach [4], neural network approach, and operator based right coprime factorization method [5] (see the references in [5]). PID control is a simple method, but it is difficult to achieve required performance when the model is not exact or there exists uncertainty of the model. The structure and parameters of the controller can be adjusted automatically by using adaptive control to satisfy control requirement. Robustness of the system can be guaranteed by sliding mode control for parameter uncertainties, internal and external disturbances. Also, neural network is applied to deal with the robust nonlinear control by using the ability of its fault tolerance and adaptive learning.
In particular, operator based robust right coprime factorization method has been proved to be a new and effective method to solve the robust control and design of the nonlinear systems [5-7]. In [5], a simple and effective condition about robust right coprime factorization, called inequality condition, was proposed for nonlinear systems with unknown bounded uncertainty and output tracking problem with different spaces of reference input and output was also discussed. Furthermore, the design problem of right coprime factorization is discussed and the robust right coprime factorization approach was extended to multi-input multioutput systems with coupling effects [6,7].

Although the problems of achieving robustness with respect to disturbances and model uncertainties are as old as feedback control itself, effective systematic methods for the robust design of nonlinear systems are still a challenging topic, especially for the potential of robust nonlinear controllers in engineering applications. In this special issue, some new developments have been discussed for uncertain or disturbed nonlinear systems. Some of the works provide specific solutions for challenging robust nonlinear control problems, for example, interdisciplinary theoretical studies combined with other approaches, control algorithm development related to fault, and applications in some new fields. Fifteen papers are devoted to these aspects. 
Some interdisciplinary theoretical studies of robust nonlinear control combined with other approaches are investigated in this special issue. Adaptive robust sliding-mode vibration control is considered for a flexible beam using piezoceramic sensor and actuator. Adaptive backstepping sliding-mode control of the electronic throttle system is included. Compound control with sliding-mode disturbance observer is also reported for DC motor system. Artificial bee colony and neural network algorithms are discussed to optimize robust controller parameters. Moreover, MPC and nonlinear compensator design combined with robust nonlinear control are reported.

Fault often occurs in modern industrial control system. This special issue also contains robust control algorithm developments related to fault. Fault-tolerant control is presented for an underwater vehicle. Attainability analysis and mathematical safety assessment are included in robust nonlinear control systems.

Finally, the readers will find some new applications in this special issue, for example, in bioreactors, in biophoton emission signal analysis of wheat varieties, and in multiobject detecting and tracking. Also, this special issue shows an application to pneumatic actuator with stiffness characteristic.

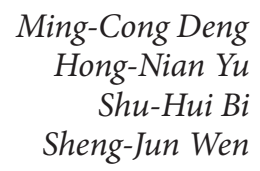

\section{References}

[1] K. J. Åström and T. Hägglund, PID Controllers: Theory, Design, and Tuning, ISA: The Instrumentation, Systems, and Automation Society, 2nd edition, 1995.

[2] A. Isidori, Nonlinear Control Systems, Springer, London, UK, 3rd edition, 1995.

[3] V. I. Utkin, "Sliding mode control design principles and applications to electric drives," IEEE Transactions on Industrial Electronics, vol. 40, no. 1, pp. 23-36, 1993.

[4] I. D. Landau, Adaptive Control: The Model Reference Approach, Marcel Dekker, New York, NY, USA, 1979.

[5] M. Deng, A. Inoue, and K. Ishikawa, "Operator-based nonlinear feedback control design using robust right coprime factorization," IEEE Transactions on Automatic Control, vol. 51, no. 4, pp. 645-648, 2006.

[6] S. Wen and M. Deng, "Operator-based robust nonlinear control and fault detection for a Peltier actuated thermal process," Mathematical and Computer Modelling, vol. 57, no. 1-2, pp. 1629, 2013.

[7] S. Bi and M. Deng, "Operator-based robust control design for nonlinear plants with perturbation," International Journal of Control, vol. 84, no. 4, pp. 815-821, 2011. 


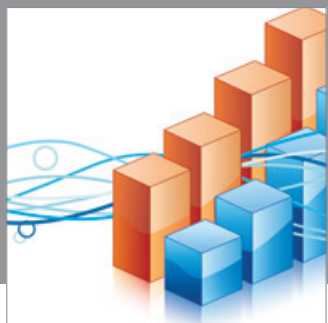

Advances in

Operations Research

mansans

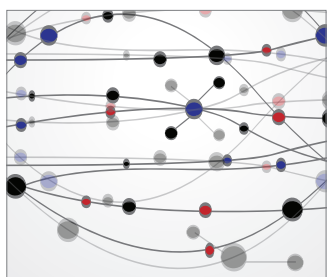

The Scientific World Journal
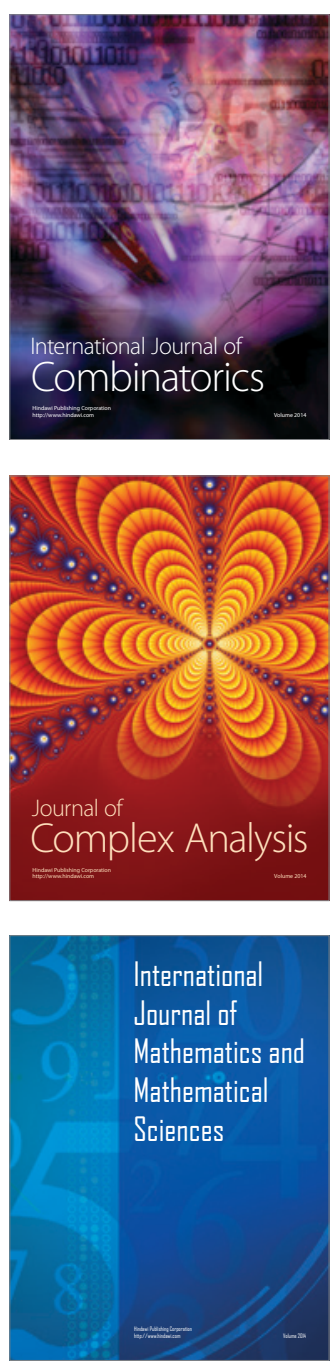
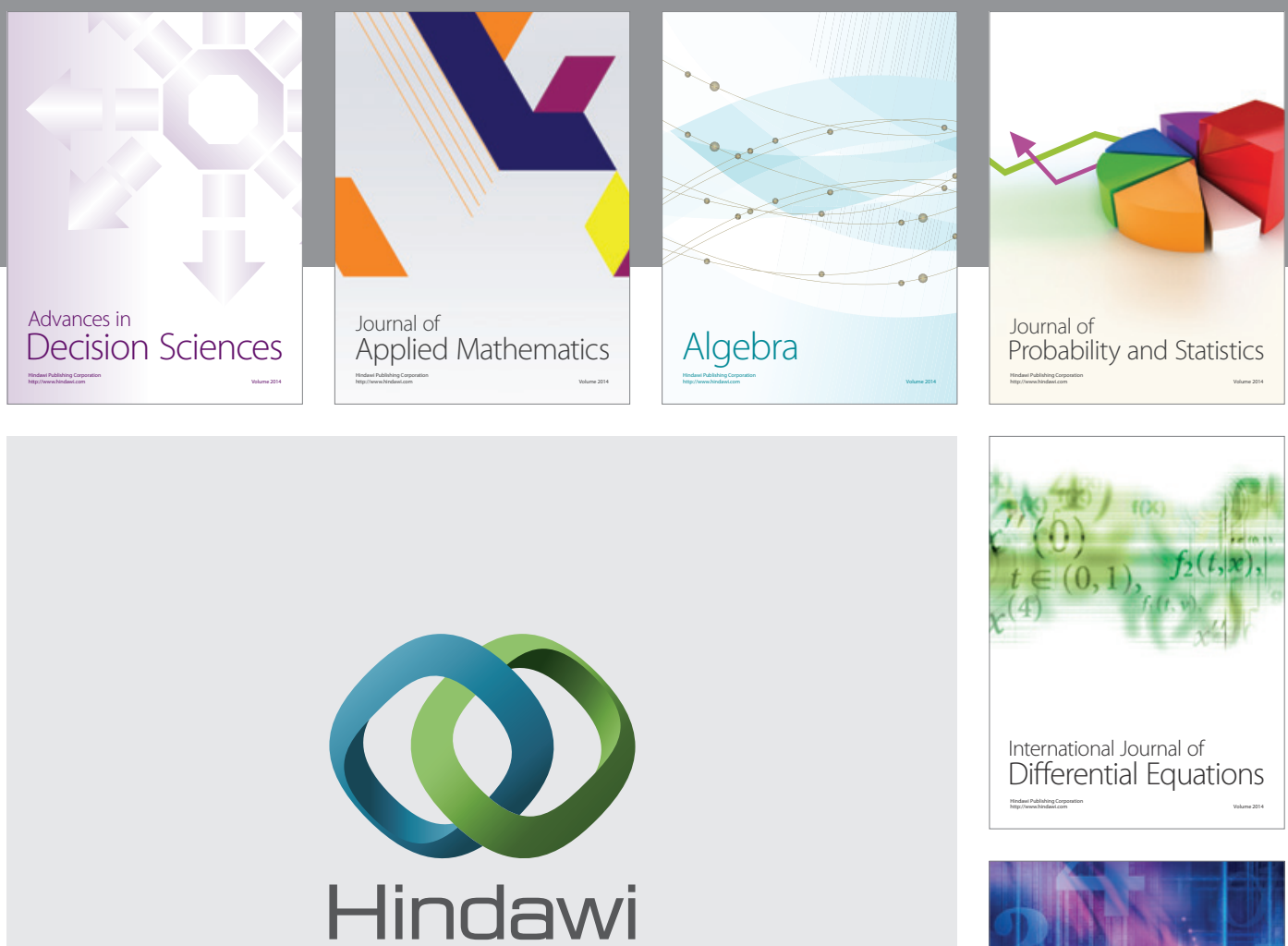

Submit your manuscripts at http://www.hindawi.com
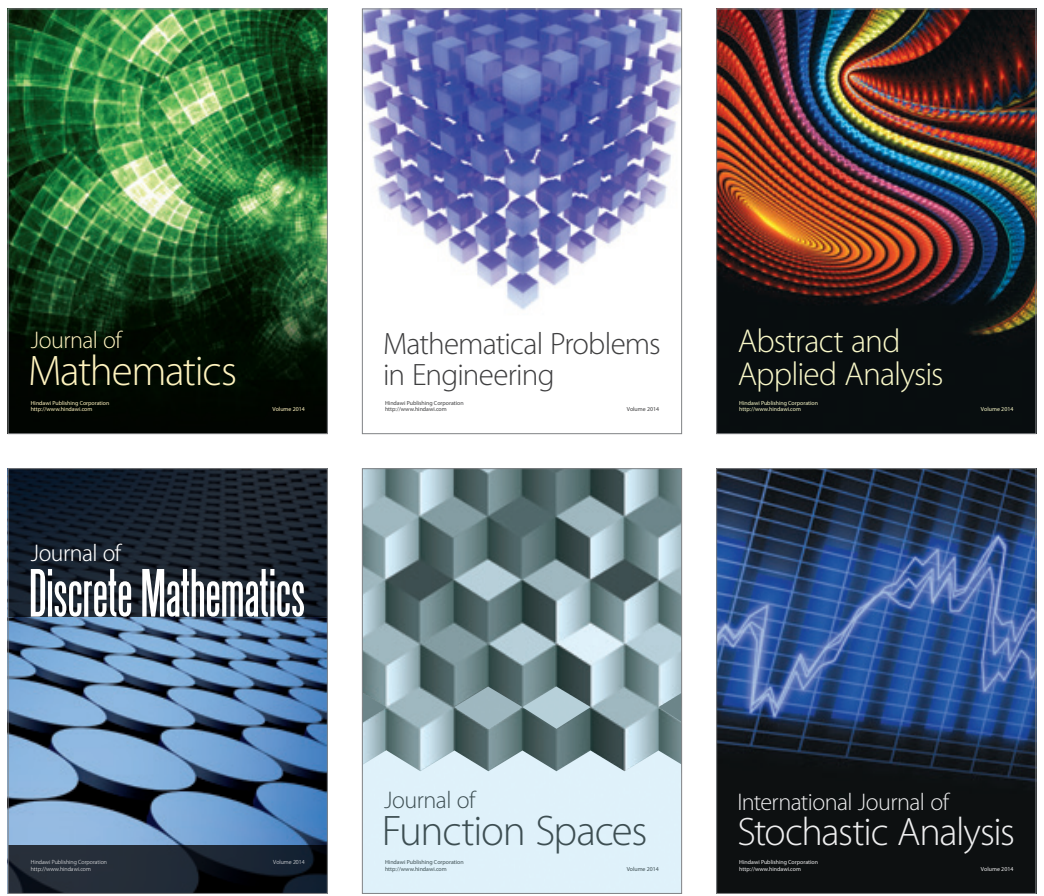

Journal of

Function Spaces

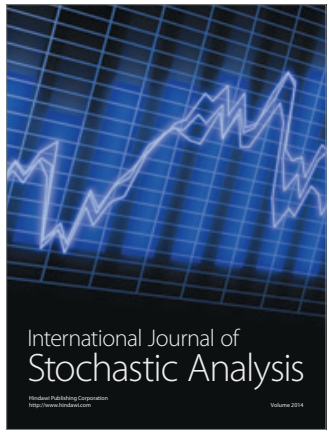

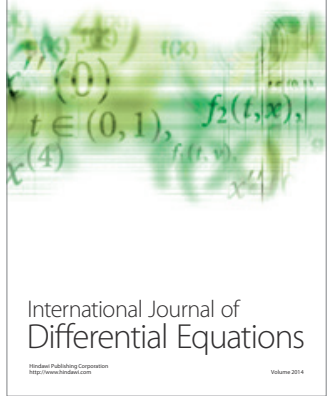
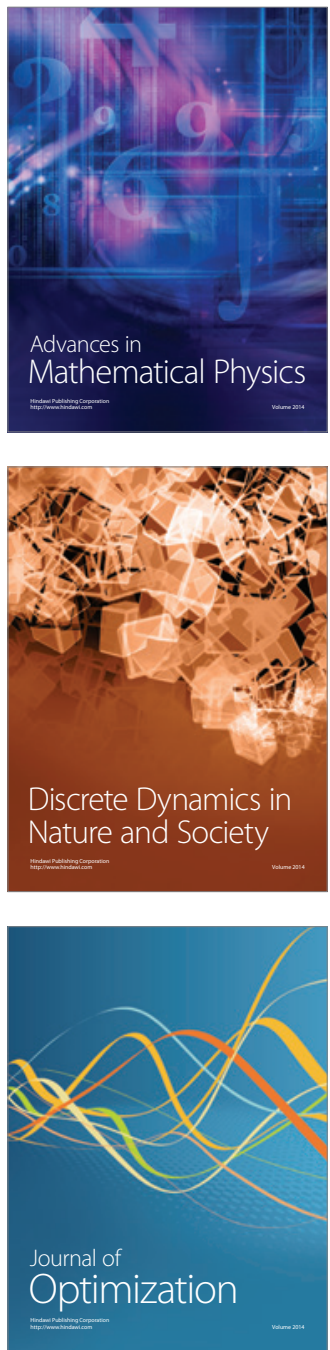